\title{
COMPARISON OF THE COBB ANGLE MEASUREMENT BETWEEN MANUAL AND DIGITAL METHODS AMONG FIVE MILITARY HOSPITALS
}

\author{
Chalermrit Kraturerk, Sompob Poopitaya, Roongrath Chitragran*
}

\author{
Spine Unit, Department of Orthopaedics, Phramongkutklao Hospital and Phramongkutklao \\ College of Medicine, Bangkok, Thailand
}

\begin{abstract}
Background: The Cobb angle measurement of the spine is an important parameter for assessing patients with scoliosis in terms of diagnosis, treatment planning, follow-up for severity and disease progression. This angle can be obtained from a plain X-ray film in an upright posteroanterior view or can be measured from digital images. A $5^{\circ}$ curve progression is considered clinically significant.

Objective: This study aimed to compare the accuracy and difference of the Cobb angle measurements between manual and digital methods among 5 military hospitals located in the $1^{\text {st }}$ area of command having orthopedic services and using different Picture Archiving and Communication (PACS).

Methods: Using plain X- ray films of patients diagnosed with scoliosis, a comparative study of the Cobb angle measurement was conducted. A total of 120 images were recorded in a compact disc in the Digital Imaging and Communications in Medicine (DICOM) system and was used to install in computer systems of 5 military hospitals (Hospitals A, B, C, D, and E), and then was interpreted using each hospital digital PACS. The mean difference of $5^{\circ}$ is considered clinically significant. The validity of measurements was analyzed using paired t- test for the mean equivalence. The reliability of one time measurement was also performed using Intraclass Correlation Coefficient (ICC).

Results: Both one time and an average of three times of digital measurements among Hospitals A, B, $\mathrm{C}, \mathrm{D}$ and E revealed significant differences when compared with the manual measurement $(p<0.01)$. However, no clinical significance of both one time and the averaged three measurements were observed when the mean difference was less than $5^{\circ}$. In the combined process group (Hospitals $\mathrm{C}, \mathrm{D}$ and $\mathrm{E}$ ), a significant difference of the manual and digital measurements was observed $(p<0.01)$. However, no clinical significance using both one time and averaged three time measurements was found when the mean difference was less than $5^{\circ}$. The data of one time digital measurements were reliable $(\mathrm{ICC}=0.9)$. Conclusion: The use of digital Cobb angle measurement is a convenient practice. A significant difference using manual and digital methods was found using both one and combined processes; however, no clinical significance was observed. One time digital measurement revealed validity as those found in three time averaged measurements.
\end{abstract}

Keywords: Scoliosis, Cobb angle, Manual measurement, Digital measurement

J Southeast Asian Med Res 2021: 5 (2):51-57

https://www.jseamed.org

Correspondence to:

Chitragran R, Department of Orthopaedics, Phramongkutklao Hospital and Phramongkutklao College of Medicine, Bangkok, Thailand

E-mail: roongrathc@hotmail.com

Received: 10 May 2021

Revised: 14 June 2021

Accepted: 2 September 2021 


\section{Introduction}

The Cobb angle measurement is the gold standard to quantitatively measure the lateral deviation of the spine and is usually considered for diagnosis, planning of management and follow-up of patients with scoliosis. This measurement is also used to determine the severity of the curve and estimate its progression which is very helpful among orthopedists especially spine surgeons for planning the most appropriate treatment. Technological advances have allowed and increased the use of digital X-ray images in clinical practices of which the PACS (Picture Archiving and Communication System) has been used in intrahospital computerized systems. This technology offers many advantages, i.e., efficient image storage, rapid data sorting, ease to access and review of studies comparing different follow-up for patients with scoliosis. Varieties of digital systems of PACS that have been designed from various companies are available in many hospitals. However, the accuracy of each digital system of PACS has never been compared among military hospitals in Thailand.

The Cobb angle measurement method has been used in clinical practices as a simple and well known technique using manual measurement. Using a pencil and a goniometer, the doctor draws lines on plain $\mathrm{X}$ - ray film in posteroanterior view and calculates the scoliotic or the Cobb angle formed by the most tilting vertebra, from the upper end vertebra to the lower end vertebra. Despite this simplicity, several factors may influence the measurement including the use of different goniometers, size and sharpness of pencils, correction of selected vertebrae and level of experiences with precision of the doctors. Thus, the main principle of digital measurement of Cobb angle is to reduce the impact of human error.

The reliability of the measurement of the Cobb angle using manual versus digital methods have been studied. Brian et al. (1) compared the Cobb angle measurement of scoliosis radiographs with preselected end vertebrae traditional versus digital acquired X-ray images. Tanure et al. (2) also studied the reliability assessment of Cobb angle measurement using manual and digital methods. The results showed that the mean error of the manual method was 3.8 degrees while the digital method was 3.6 degrees. Many related studies have reported mean error ranging from 1.7 degree to 6.5 degrees. ${ }^{(3-6)}$ However, in military hospitals, the efficacy of the digital X-ray system for the Cobb angle measurement used in multi center hospitals comprising different brands of PACS has never been compared. Due to multidisciplinary management of scoliosis, the X-ray films have been interpreted by different individuals. Decisions regarding surgical interventions and physiotherapeutic or orthotic options can be made based on scoliotic curve progression denoted by the Cobb angle. Hence an accurate, precise, and reliablemethod is ofparamountclinical importance, especially considering even a 5 degree curve progression is potentially clinically different. ${ }^{(7)}$

Therefore, to demonstrate the reliability and accuracy of a variety of digital methods in multi center hospitals compared with a traditional manual method, the digital measurements among five military hospitals in the first area of command having different PACS were compared and reported in detail for each system.

\section{Methods}

To calculate the sample size according to the study of Tanure et al. (2), different measurements of the Cobb angle were $3.85 \pm 3.45$ degrees using the manual method while those obtained by the digital method were $3.61 \pm 3.18$ degrees, a minimum of 118 plain X-ray films were necessary to provide a $95 \%$ confidence interval at a margin of error of $5 \%$. This study was approved by the Institutional Review Board, Medical Department, Royal Thai Army (IRBRTA 2190/2561).

In this study, a total of 120 plain X-ray films from patients receiving a diagnosis of scoliosis, both idiopathic and degenerative type, were available to assess the validity and reliability of the digital method to assess the Cobb angle. These plain X-ray films were retrospectively randomized chosen from the database of patients with scoliosis attending Phramongkutklao Hospital from 2012 to 2018. The X-ray films were without predilection of age, sex, location, type, or magnitude of the curvature. They were all posteroanterior views of standing whole spine films within 36 inches long. 
All 120 images then were recorded in a compact disc in the Digital Imaging and Communications in Medicine (DICOM) system. This compact disc was used to install in five military hospitals computer systems and then was interpreted by each hospital's digital PACS. The study population magnitude of the curve ranging from the smallest one ( 5 degrees) to a largest one ( 80 degrees). Each chosen curvature was recorded with preselected end vertebrae to prevent human error from measurement. We endeavored to locate and choose the most obvious margin of the end plates for the upper and lower vertebrae of each plain film. The PACS were: UniWeb viewer in Hospital A, VUE Solutions in Hospital B, medSynapse (former) in Hospital C, medSynapse (new) in Hospital $\mathrm{D}$ and TRACS DICOM VIEWER in Hospital E.

Manual and digital measurements were compared as follows: 1) a single pair of each hospital (manual vs. digital measurements conducted in Hospitals A, B, C, D and E) using only the one time measurement; 2) manual vs. digital measurements conducted in Hospitals A, $\mathrm{B}, \mathrm{C}, \mathrm{D}$ and $\mathrm{E}$ using the average of three time measurements; 3) manual vs. a single process of measurement groups (Hospitals A and B) both one time and the average of three time measurements; 4) manual vs. combined process of measurement groups (Hospitals C, D and E) both one time and the average of three time measurements; 5) manual vs. subset of combined process of measurement (linear tips; Hospital C and circle tips; Hospital E ) both one time and the average of three time measurements.

Only one person used the traditional (manual) method as well as the digital (software based) method to assess scoliotic images obtained from 120 patients.

\section{Manual measurement}

One spine fellow performed the Cobb measurement technique using preselected end vertebrae of each plain film obtained from 120 patients. A pencil size HB with sharp pencil lead (for precision of lining) was used to make a straight line. A goniometer then was used to calculate the Cobb angle. To make a precise line and avoid scratches on the X-ray films, all 120 curves were recorded in a set of manual methods using only one time measurement (Figure1).

\section{Digital measurement}

The compact disc in the DICOM system containing the same images of all 120 patients was provided for digital measurements using the PACS of each military hospital (Hospitals A, B, C, D and E), located at the first area of command. Each hospital data set was individually recorded at the first time measurement as well as the average (mean) of three time measurements. The three time measurements were used to compare with one time measurements.

The differences of measurement processes among these digital PACS were grouped. The first group was a single step measurement, which was used in Hospitals A and B. This single step of measurement of the Cobb angle was automatically calculated and interpreted when only two lines were drawn at both upper and lower end plates. (Figure 2)

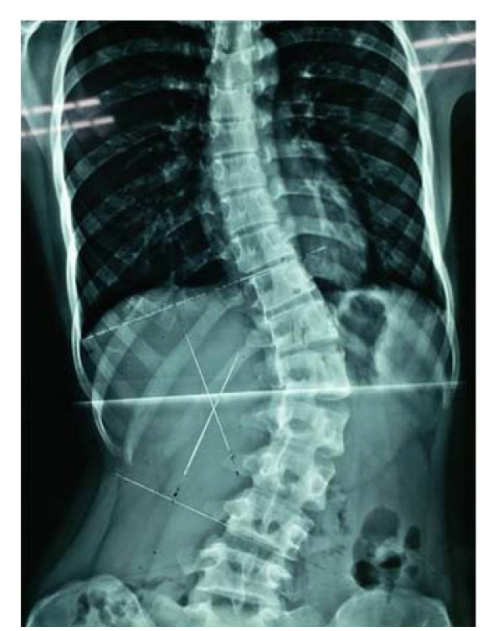

Figure 1. The Cobb angle using a manual measurement 


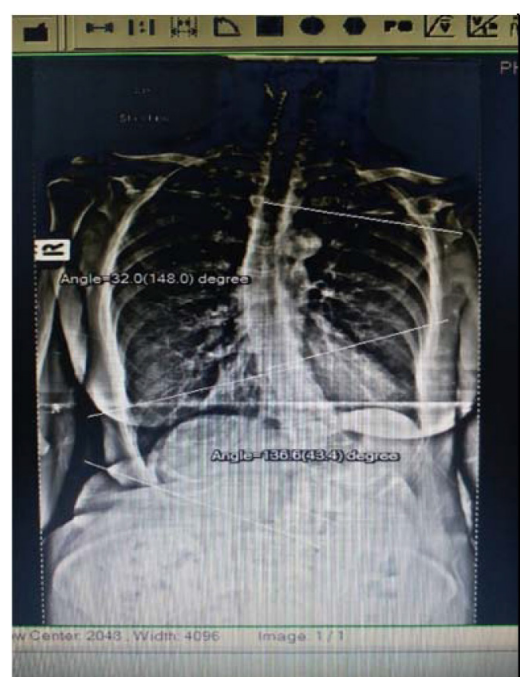

Hospital A

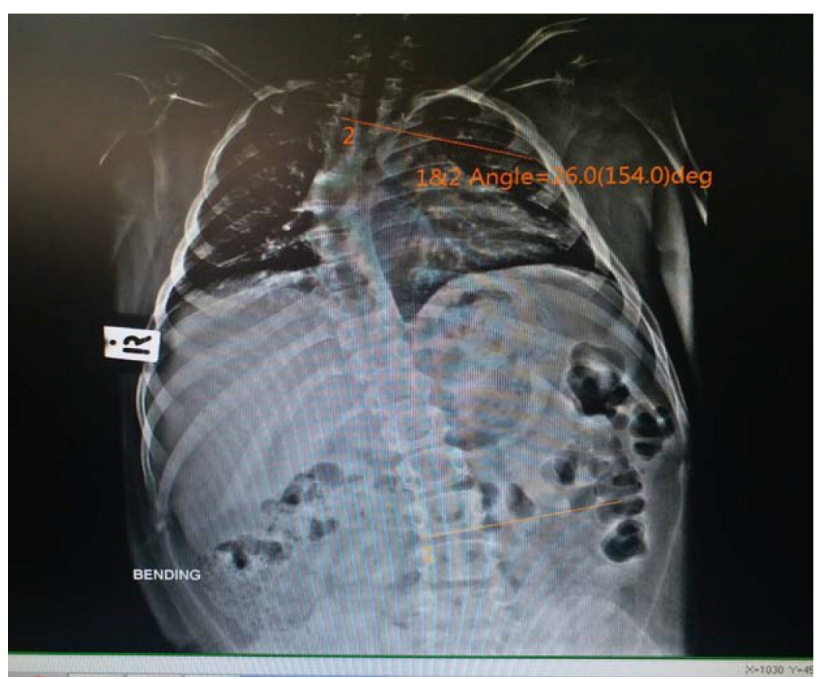

Hospital B

Figure 2. Cobb angle performed at Hospitals A and B using digital measurements.

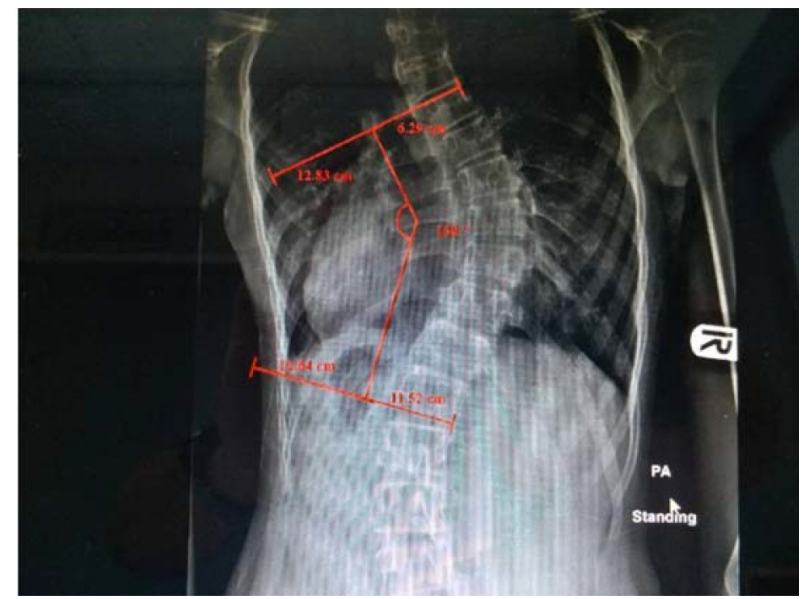

Hospital C

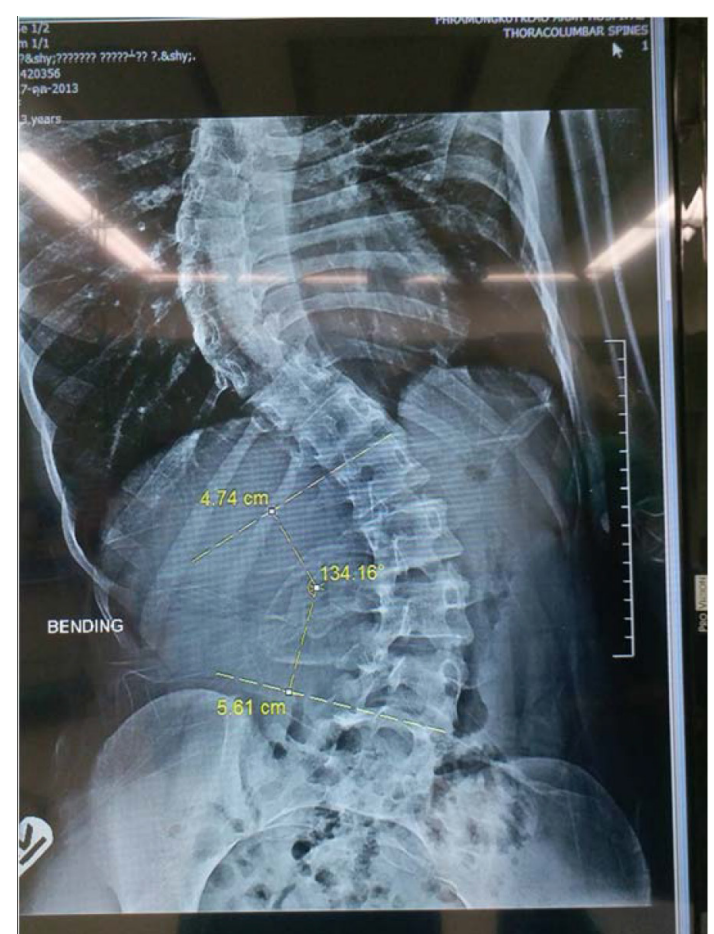

Hospital D

Figure 3. Cobb angle performed at Hospitals C and D using digital measurements

The second group was a combined step of measurements which was used in Hospitals C, $\mathrm{D}$ and E. The Cobb angle was automatically calculated and interpreted when two lines were drawn at both upper and lower end plates at first and the angle joining line was drawn in the following part.

Moreover, the combined step of the measurement group differed in some details of the angle joining lines. The Cobb angle performed in
Hospitals $\mathrm{C}$ and D used a linear tip on the angle joining line (Figure 3) while Hospital E used circle tips (Figure 4).

\section{Statistical analysis}

Validity and reliability of Cobb angle measurements using manual and digital methods were assessed to compare intra-observer differences. The analysis of intra-observer variation calculated for each data set was expressed in Mean \pm SD. 
A 5 degree curve progression was considered clinically significant. ${ }^{(7)}$ Using the paired $-t$ test for the mean equivalence when the difference of measurement was less than 5 degrees, a $p$-value less than $<0.05$ was considered statistically significant measurements. The reliability of measurements was tested using the Intraclass Correlation Coefficient (ICC).

\section{Results}

The mean $\pm \mathrm{SD}$ of one time measurement of the Cobb angle using manual and digital measurements conducted at five military hospitals (Hospitals A, B, C, D and E) was demonstrated. The intra-observer analysis and validity of only one measurement is shown in Table 1. All digital measurement data revealed significant differences when compared with the manual measurement

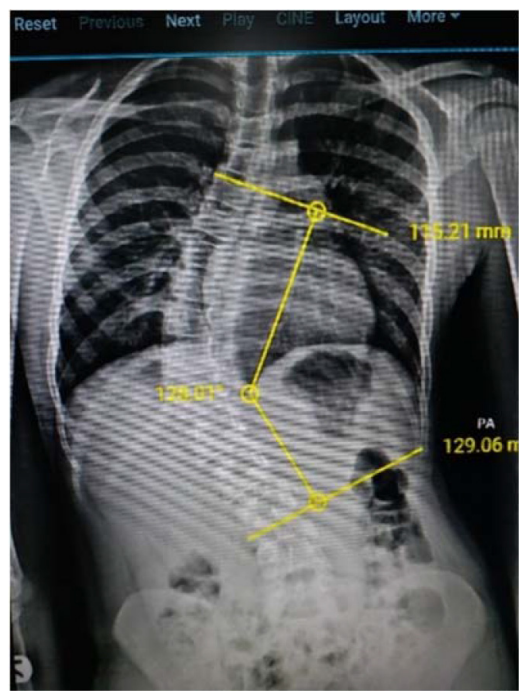

Figure 4. Cobb angle performed at Hospital E using digital measurement

Table 1. Comparison of the Cobb angle using manual and digital measurements conducted in 5 hospitals.

\begin{tabular}{lccc}
\hline $\begin{array}{c}\text { Comparison } \\
\text { Manual VS Digital } \\
\text { Measurements }\end{array}$ & $\begin{array}{c}\text { The Cobb angle } \\
(\text { Mean } \pm \text { SD) }\end{array}$ & $\begin{array}{c}\text { Paired differences of the Cobb angle } \\
\text { between manual VS digital } \\
\text { measurements (Mean } \pm \text { SD) }\end{array}$ & $p$-value \\
\hline Manual measurement & $31.68 \pm 16.54$ & $1.15 \pm 4.76$ & $<0.001$ \\
\hline Hospital A & $32.84 \pm 17.10$ & $0.72 \pm 3.81$ & $<0.001$ \\
Hospital B & $32.40 \pm 16.36$ & $1.58 \pm 4.85$ & $<0.001$ \\
Hospital C & $33.26 \pm 15.80$ & $1.52 \pm 4.25$ & $<0.001$ \\
Hospital D & $33.20 \pm 16.62$ & $3.97 \pm 3.87$ & $<0.001$ \\
Hospital E & $35.65 \pm 16.59$ & $0.93 \pm 3.83$ & 0.009 \\
Hospitals A and B & $32.62 \pm 16.62$ & $2.35 \pm 3.71$ & $<0.001$ \\
Hospitals C, D and E & $34.04 \pm 16.18$ & $1.79 \pm 3.48$ & $<0.001$ \\
Hospitals A, B, C, D and E & $33.47 \pm 16.30$ & &
\end{tabular}

Using the paired $-\mathrm{t}$ test for mean equivalence, $p$-value less than $<0.05$ was considered statistically significant. Mean differences between the two methods not more than 5 degrees was considered not clinically significant. 
Table 2. Reliability of one time measurement of manual and digital measurements using Intraclass Correlation Coefficient (ICC)

\begin{tabular}{ccccc}
\hline Hospital & ICC & \multicolumn{2}{c}{$95 \%$ CI } & $p$-value \\
\cline { 3 - 4 } & & Lower & Upper & \\
\hline A & 0.980 & 0.971 & 0.986 & $<0.001$ \\
B & 0.986 & 0.980 & 0.991 & $<0.001$ \\
C & 0.977 & 0.967 & 0.984 & $<0.001$ \\
D & 0.983 & 0.976 & 0.988 & $<0.001$ \\
E & 0.986 & 0.980 & 0.990 & $<0.001$ \\
A and B & 0.986 & 0.981 & 0.991 & $<0.001$ \\
C, D and E & 0.987 & 0.981 & 0.991 & $<0.001$ \\
A, B, C, D and E & 0.989 & 0.984 & 0.992 & $<0.001$ \\
\hline
\end{tabular}

$P$-value less than 0.05 was statistically significant.

Table 3. Comparison of the Cobb angle using manual and averaged three digital measurements conducted in 5 hospitals (single and combined measurements)

\begin{tabular}{lccc}
\hline \multicolumn{1}{c}{$\begin{array}{c}\text { Comparison } \\
\text { Manual VS Digital } \\
\text { Measurement }\end{array}$} & $\begin{array}{c}\text { The Cobb angle } \\
\text { (Mean } \pm \text { SD) }\end{array}$ & $\begin{array}{c}\text { Paired Differences of the Cobb } \\
\text { angle between manual VS } \\
\text { digital measurement (Mean } \pm \text { SD) }\end{array}$ & $p$-value \\
\hline Manual measurement & $31.68 \pm 16.54$ & $1.55 \pm 4.15$ & $<0.001$ \\
Hospital A & $33.23 \pm 16.20$ & $0.74 \pm 4.30$ & $<0.001$ \\
Hospital B & $32.42 \pm 16.39$ & $1.04 \pm 2.77$ & $<0.001$ \\
Hospital C & $32.73 \pm 16.08$ & $1.58 \pm 3.89$ & $<0.001$ \\
Hospital D & $33.27 \pm 16.78$ & $3.85 \pm 3.26$ & $<0.001$ \\
Hospital E & $35.53 \pm 16.67$ & $1.14 \pm 3.36$ & $<0.001$ \\
Hospitals A and B & $32.83 \pm 16.09$ & $2.16 \pm 2.67$ & $<0.001$ \\
Hospitals C, D and E & $33.84 \pm 16.39$ & $1.75 \pm 2.62$ & $<0.001$ \\
Hospitals A, B, C, D and E & $33.44 \pm 16.21$ & & \\
\hline
\end{tabular}

Using the paired -t test for mean equivalence, $p$-value less than $<0.05$ was considered statistically significant. Mean difference between the two methods not more than 5 degrees was considered not clinically significant.

data $(p<0.01)$. However, digital measurements among Hospitals A B, C, D and E revealed no clinical significance within 5 degree variation (Tables 1 and 3). All measurements were reliable according to the Intraclass Correlation Coefficient (ICC $=0.9$ ) as shown in Table 2.

For averaged three time measurements, similar results were observed as only one time measurement (Table 3).

\section{Discussion}

Although the Cobb angle is an objective 2-dimensional measure of a 3D spinal deformity, and despite its high intra and inter-observer error, it still remains the "gold standard" radiographic parameter to quantify scoliotic curves due to its inherent simplicity. Phramongkutklao Hospital, a tertiary military hospital caring for multi disciplinary treatment of scoliosis, has transferred 
patients with scoliosis from other military hospitals. Thus, assessing patients with scoliosis and treatment planning based on the degree of spinal curve measured by the Cobb angle method is important. Traditionally, the manual measurement has been used before the advent of computerized programs. Currently, the PACS has conveniently been used to digitally measure the Cobb angle. Usually, according to Tanure et. al $^{(2)}$, five-degree variations are acceptable having the mean error range from 1.7 to 6.5 degrees. In this study, the manual measurement was used as the reference method to compare with five digital measurement systems. Due to varieties of PACS used among military hospitals, comparisons were divided in two groups. The first group had a single process of digital measurement that used only two lines at both endplates and then the Cobb angle was automatically calculated. The second group used a combined process where the Cobb angle could be automatically calculated after the perpendicular line was applied to both endplate lines. This second group differed in their tools and tip of the perpendicular line. Normally, the perpendicular line has a straight linear line that is easy to precisely apply to the endplate line. In Hospital E, they used the circle tip of the perpendicular line which made the measurement contain more error than that of a linear line. The single process digital measurement (Hospitals A and B) showed significant difference compared with those obtained from the manual method but remained in acceptable ranges with no clinical differences compared with the traditional methods. The combined process of digital measurements of Hospitals C, D and E also showed significant difference where no clinical significance was observed. Additionally, our study showed that the Cobb angle using both one time measurement and average three time measurements was practical. One aspect considered was how many times the Cobb angle should be measured to obtain the most accurate result. Our study revealed that only one time measurement was reliable and quite practical that the result was compatible with the value of both manual and three-time digital measurement methods.

\section{Conclusion}

The use of a digital Cobb angle measurement is a convenient practice. A significant difference using the manual and digital methods was found using both one and combined process; however, no clinical significance was observed. One time digital measurement revealed validity similar to those found in three time average measurements. Thus, one time digital measurement using the PACS can be used for the Cobb angle.

\section{References}

1. Brian W, Joshua A. Comparison of Cobb angle measurement of scoliosis radiographs with preselected end vertebrae : traditional versus digital acquisition. Spine 2007: 98-105

2. Tanure MC, Pinheiro AP, Oliviera AS. Reliability assessment of Cobb angle measurement using manual and digital methods. Spine J 2010; 10: 769-7

3. Vrtovec T, Pernus F, Likar B. A review of method for quantitative evaluation of spinal curvature. Eur Spine J 2009; 18: 593-607

4. Shea KG, Steven PM, Nelson M, Smith JT, Masters KS, Yandow S. A comparison of manual versus computer-assisted radiographic measurement: intraobserver measurement variability for Cobb angle. Spine 1998; 23: 551-5

5. Ricart PA, Andres TM, Apacidis A. Validity of Cobb angle measurements using digitally photographed radiographs. Spine J 2011; 11: 942-46.

6. Chockalingham N, Dangerfield PH, Giakas G, Cochrane T, Dorgan JC. Computer assisted Cobb measurement of scoliosis. Eur Spine J 2002; 11: 353-7.

7. Bunnell WP. Selective screening for scoliosis. Clin Orthop Relat Res 2005; 434: 40-45 